ched and (optionally) their present occupation. All this information should also be given in one of the official languages of the ISA (English, French, Spanish).

6. Initially, a jury will consider which papers reach a sufficiently high standard to be issued with a letter of official commendation and be listed on ISA website. Each language jury will then preselect (by September 2005) a maximum of three papers. These finalists will receive Merit Award Certificates, a four-year membership in the ISA, and an invitation to participate in the XVI World Congress. The ISA, however, cannot guarantee to cover their travel costs. All authors thus preselected will also be invited to participate in a one-week seminar prior to the Congress.

Out of the preselected finalists, a Grand Jury chaired by the ISA President Piotr Sztompka will select up to five winning papers. Their authors will be immediately invited, all expenses paid, to participate in the World Congress. In case of multiple authorship, the subvention will have to be shared.

Additional information may be obtained from the Competition Secretariat in Milton Keynes.

\title{
Gutachterinnen und Gutachter der KZfSS 2002/2003
}

An der Begutachtung von Manuskripten, die der Zeitschrift im Zeitraum zwischen dem 1. Juli 2002 und dem 30. Juni 2003 eingereicht wurden, haben die folgenden Kolleginnen und Kollegen mitgewirkt. Wir danken ihnen sehr herzlich für die Beteiligung an der Entstehung der Zeitschrift.

\author{
Beckert-Zieglschmid, Claudia (Leipzig) \\ Blasius, Jörg (Bonn) \\ Brettschneider, Frank (Augsburg) \\ Breuer, Stefan (Hamburg) \\ Bulmahn, Thomas (Strausberg) \\ Brose, Hanns-Georg (Duisburg) \\ Büchel, Felix (Berlin) \\ Dülmer, Hermann (Köln) \\ Ebbinghaus, Bernhard (Köln) \\ Epp, Astrid (Bielefeld) \\ Fleck, Christian (Graz) \\ Geißler, Rainer (Siegen) \\ Habich, Roland (Berlin) \\ Hartmann, Michael (Darmstadt) \\ Hartmann, Peter H. (Düsseldorf) \\ Heidenreich, Martin (Bamberg) \\ Hoffmeyer-Zlotnik, Jürgen (Mannheim) \\ Höpner, Martin (Köln) \\ Immerfall, Stefan (Schwäbisch-Gmünd) \\ Kalter, Frank (Mannheim) \\ Klein, Markus (Köln) \\ Konietzka, Dirk (Rostock) \\ Kopp, Johannes (Landau)
}

\author{
Lange, Elmar (Bielefeld) \\ Lüdemann, Christian (Bremen) \\ Mangold, Werner (Erlangen) \\ Müller-Schneider, Thomas (Bamberg) \\ Neidhardt, Friedhelm (Berlin) \\ Ohr, Dieter (Köln) \\ Onnen-Isemann, Corinna (Regensburg) \\ Podsiadlowski, Astrid (Gräfelfing) \\ Porst, Rolf (Mannheim) \\ Quandt, Markus (Köln) \\ Raub, Werner (Utrecht) \\ Reichertz, Jo (Essen) \\ Rosar, Ulrich (Köln) \\ Rohwer, Götz (Bochum) \\ Sackmann, Reinhold (Bremen) \\ Schmid, Michael (Augsburg) \\ Schnell, Rainer (Konstanz) \\ Trommsdorff, Gisela (Konstanz) \\ Wiener, Bettina (Halle/Saale) \\ Windolf, Paul (Trier) \\ Wobbe, Theresa (Erfurt) \\ Zündorf, Lutz (Lüneburg)
}

